\title{
Optimal reduced dimensional representation of classical molecular dynamics
}

Bijoy K. Dey and H. RabitzAttila Askar

Citation: The Journal of Chemical Physics 119, 5379 (2003); doi: 10.1063/1.1599349

View online: http://dx.doi.org/10.1063/1.1599349

View Table of Contents: http://aip.scitation.org/toc/jcp/119/11

Published by the American Institute of Physics

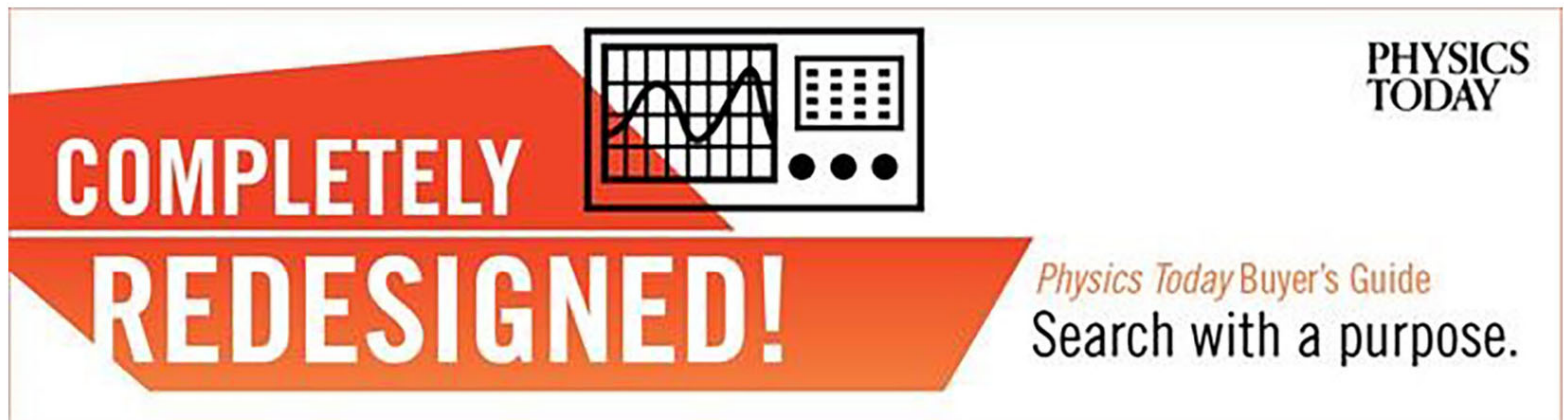




\title{
Optimal reduced dimensional representation of classical molecular dynamics
}

\author{
Bijoy K. Dey and H. Rabitz \\ Department of Chemistry, Princeton University, Princeton, New Jersey 08544 \\ Attila Askar \\ Mathematics Department, Koc University, Istinye, Istanbul, Turkey 80860
}

(Received 27 January 2003; accepted 18 June 2003)

\begin{abstract}
An optimal reduced space method for capturing the low-frequency motion in classical molecular dynamics calculations is presented. This technique provides a systematic means for carrying out reduced-dimensional calculations in an effective set of reduced coordinates. The method prescribes an optimal reduced subspace linear transformation for the low frequency motion. The method is illustrated with a dynamics calculation for a model potential, where the original six-dimensional space is reduced to two (three) dimensions, depending on the desired frequency cutoff value. (C) 2003 American Institute of Physics. [DOI: 10.1063/1.1599349]
\end{abstract}

\section{INTRODUCTION}

Classical molecular dynamics is an important tool in chemistry for many applications, e.g., proteins. ${ }^{1,2}$ Such dynamical studies are computationally time consuming because of the large number of degrees-of-freedom and the coupling amongst them. Experience suggests that all of the degreesof-freedom or all of the frequency components will not be equally important for most dynamical processes. Simulation times out to a few nanoseconds are much shorter than the time scales of many important conformational processes and this situation poses a major obstacle limiting the usefulness of classical molecular dynamics simulations. Many attempts, ${ }^{3-21}$ aimed at containing the system dimensions have been made over the years to aid in the effort to extend the time scale of the simulations, but as yet, no fully satisfactory solution has been found.

One approach ${ }^{5,6}$ is principal component analysis (PCA), also known as the Karhunen-Loeve expansion in time series analysis. The method has been employed with the hope of describing molecular dynamics trajectories in terms of a small number of variables or essential degrees of freedom. ${ }^{7,9,10,22-24}$ Here one determines a small number of important modes by PCA and projects the equation of motion on the resulting low dimensional vector space. This method has been successful in treating hydrodynamic systems, ${ }^{11}$ but not as satisfactory in molecular dynamics simulations. ${ }^{25}$

Another way in which the dimensionality may be reduced is by neglecting some degrees of freedom so that the corresponding motions are not present. Some dynamical processes can be modeled quite well in this way. For example, intramolecular vibrational energy relaxation for the overtones of planar benzene can be well described by a four-atom model. ${ }^{12}$ However, such models are often oversimplified. A somewhat higher level of sophistication of this same idea has been suggested, ${ }^{13-16}$ and constrained classical dynamics approaches have also been introduced. ${ }^{17-21}$ For example, in an application ${ }^{19}$ to the classical motion of a polyatomic system with internal coordinate constraints, the unconstrained positions were calculated first, and then the constraint equations were solved for the undetermined multipliers from which the displacements were obtained. An application of this idea was performed $^{21}$ for the intramolecular dynamics of HONO.

Filters may be used for removing the unwanted frequencies from signals. ${ }^{26-28}$ A proposal along these lines used a filter to remove the high frequency motion from a set of stored atomic coordinates, allowing the important motions within the simulation to be observed more easily. ${ }^{29}$ The wavelet transform has also been used ${ }^{30}$ in the analysis of molecular dynamics trajectories. The Fourier transform basis set is localized in frequency but the wavelet basis set is localized in time as well, resulting in resolution of time localized rare events. Besides filtering and constraint-based reduction of dimensionality, another approach consists in altering the potential energy function by adding extra forces ${ }^{31}$ or by a parametric additive potential correction term. ${ }^{32}$ By properly choosing the additive term the trajectories can be smoothed by removing unwanted oscillations.

In general, the amplitude associated with any degree of freedom will typically depend on many, possibly all, of the degrees-of-freedom of the system. We seek a representation of the dynamics of a system with $N$-degrees of freedom in terms of a surrogate system with fewer degrees of freedom. Along these lines ${ }^{33,34}$ previous works introduced the notion of subspace dynamics. The subspace was selected from the eigenvectors of the locally linearized system based on a high frequency cutoff of the spectrum. These eigenvectors were used to project the full dynamical equations into the subspace leading to a small number of effective dynamical equations of motion for the system. Recently, a reduced space dynamical method based on orthogonal decomposition of the eigenmodes has been successfully applied to treat fluid dynamics problems. ${ }^{35,36}$

This paper studies the subspace method ${ }^{33,34}$ more systematically based on optimally designing the subspace where the original set of dynamical equations are projected onto another reduced set of effective equations. We use a genetic algorithm to identify the subspace. This is a general approach for reducing the dimensionality of a system, effectively fil- 
tering out the unwanted high frequency parts of the collective dynamics and thereby permitting an exploration of the low frequency average properties of the system. The present paper aims to present the basic concept of seeking an optimal low dimensional subspace for long time dynamical motion. Adapting the concept to become an efficient numerical algorithm will be dealt with in later works.

Section II describes the general formalism of the method. Application of the method to a model system is described in Sec. III. Numerical results obtained with the model are presented in Sec. IV while Sec. V concludes the paper.

\section{FORMALISM}

\section{A. Subspace dynamics}

Consider Newton's equations for a molecular system with $N$ degrees of freedom,

$$
\begin{aligned}
& \ddot{\mathbf{q}}=-\mathbf{h}(\mathbf{q}), \\
& \mathbf{q}(0)=\mathbf{q}_{0}, \\
& \dot{\mathbf{q}}(0)=\dot{\mathbf{q}}_{0},
\end{aligned}
$$

where $\mathbf{q}$ is an $N$-dimensional vector $\mathbf{q} \equiv\left(q_{1}, q_{2}, \ldots, q_{N}\right)$ representing the positions of the particles in the system and $\mathbf{h}$ is the vector representing system force with components given by

$$
h_{i}(\mathbf{q})=\frac{\partial V(\mathbf{q})}{\partial q_{i}}
$$

$\mathbf{q}_{0}$ and $\dot{\mathbf{q}}_{0}$ denote, respectively, the initial conditions for the position and velocity vectors and $V(\mathbf{q})$ is the potential energy of the system. Here we assume unit mass of the particles in the system; the coordinates may be mass scaled to have this form.

The subspace method aims to determine an $M$-dimensional vector, $\mathbf{z}$, arising from a projection of the vector, $\mathbf{q}$,

$$
\mathbf{z}=\mathbf{P}^{T} \mathbf{q},
$$

where $\mathbf{P}$ is a time-independent $N \times M$ projector with the aim that $M \ll N$. The projector satisfies $\mathbf{P}^{T} \mathbf{P}=\mathbf{I}_{M}$ but $\mathbf{P P}^{T} \neq \mathbf{I}_{N}$, with $\mathbf{I}_{M}$ being the $M \times M$ dimensional identity matrix. In practice the true vector $\mathbf{q}$ will not be known, and the relation $\mathbf{q} \approx \mathbf{P z}$ will define the approximate positions of the particles in the system determined from the reduced space dynamical vector $\mathbf{z}$ and the transformation $\mathbf{P}$. The transformation is chosen appropriately so that the reduced system

$$
\begin{aligned}
& \ddot{\mathbf{z}}=-\mathbf{P}^{T} \mathbf{h}(\mathbf{P z})=-\mathbf{g}(\mathbf{P z}), \\
& \dot{\mathbf{z}}=\mathbf{P}^{T} \dot{\mathbf{q}}
\end{aligned}
$$

accurately reproduces the desired aspects of dynamics in the original system while $M \ll N$. The effective force $\mathbf{g}$ in the subspace is a function of the projected coordinate vector $\mathbf{z}$ and is given approximately by

$$
g_{i}(\mathbf{z})=\left.\frac{\partial}{\partial z_{i}} V(\mathbf{q})\right|_{\mathbf{q}=\mathbf{P z}} .
$$

The reduced dynamical equations generally are approximate as the relation $\mathbf{q} \approx \mathbf{P z}$ is used in Eqs. (6) and (8). The particular form of $\mathbf{g}$ above implies that the projected equation [Eq. (6)] is strictly energy conserving with the potential $V(\mathbf{z})=\left.V(\mathbf{q})\right|_{\mathbf{q}=\mathbf{P z}}$ being a function of $\mathbf{z}$. Equation (6) is equivalent to applying Lagrangian constraints with the displacements along the $N-M$ omitted collective coordinates constrained to zero. This means that the transformation $\mathbf{P}^{T}$ projects the system forces along the $M$ slow collective coordinates by not allowing the forces to act on the omitted ones. This analysis produces a new conservative Hamiltonian system, with reduced phase space Hamiltonian $H(\mathbf{z}, \dot{\mathbf{z}})$ $=\Sigma_{l} 1 / 2 \dot{z}_{l}^{2}+V(\mathbf{z})$. The resultant Hamiltonian dynamics conserves the probability density in the subspace as required by the corresponding subspace Liouville equation. The integration of the projected equation [Eq. (6)] with the initial conditions $\mathbf{z}(0)=\mathbf{P}^{T} \mathbf{q}(0), \dot{\mathbf{z}}(0)=\mathbf{P}^{T} \dot{\mathbf{q}}(0)$ yields the dynamics of $\mathbf{z}$. From $\mathbf{z}$ the dynamics of $\mathbf{q}$ can be retrieved by the back transformation $\mathbf{q} \approx \mathbf{P z}$.

\section{B. Optimal identification of the subspace}

The utility of subspace dynamics described above depends on the evaluation of the matrix $\mathbf{P}$. Here we seek an optimal identification of such a subspace. Since $\mathbf{P P}^{T} \neq \mathbf{I}$, $\mathbf{q} \neq \mathbf{P z}$, thus we define the error vector $\boldsymbol{\epsilon}$ as $\boldsymbol{\epsilon}=\mathbf{q}-\mathbf{P z}$, which gives

$$
\ddot{\boldsymbol{\epsilon}}=-(\mathbf{h}(\mathbf{q})+\mathbf{P} \ddot{\mathbf{z}}) .
$$

Here the elements of the matrix $\mathbf{P}$ are independent of time. A more general formalism, i.e., where $\mathbf{P}$ is both time and/or space dependent will be dealt in a separate paper. We desire to minimize $\|\ddot{\boldsymbol{\epsilon}}\|$ over the volume spanned by the reduced space dynamics. The minimization of $\|\ddot{\boldsymbol{\epsilon}}\|$ will be with respect to $\mathbf{P}$ producing the best transformation for any representative trajectory in the reduced subspace. A vector $\boldsymbol{\kappa}$ in either the $N$ - or $M$-dimensional space will be defined to have the norm $\|\boldsymbol{\kappa}\|^{2}=\sum_{i} \kappa_{i}^{2}$. We define a cost function $J^{(1)}$ as follows:

$$
\begin{aligned}
J^{(1)} & =\int\|\mathbf{P} \ddot{\mathbf{z}}+\mathbf{h}(\mathbf{q})\|^{2} d^{M} \mathbf{z} \\
& =\int\|\ddot{\boldsymbol{\epsilon}}\|^{2} d^{M} \mathbf{z}=\sum_{l} \int \ddot{\boldsymbol{\epsilon}}_{l}^{2} d^{M} \mathbf{z},
\end{aligned}
$$

where the integration is over the volume $\Omega_{z}$ in the reduced space and $d^{M} \mathbf{z}=d z_{1} d z_{2} \cdots d z_{M}$. Using Eqs. (6) and (7) this can be rewritten as (see Appendix A)

$$
J^{(1)}=\int\left[\|\mathbf{h}(\mathbf{q})\|^{2}-\|\mathbf{g}(\mathbf{z})\|^{2}\right] d^{M} \mathbf{z} .
$$

The cost function $J^{(1)}$ represents the difference between the norm-square forces in the approximated full space and the effective force in the subspace. In evaluating Eq. (11) the relation $\mathbf{q}=\mathbf{P z}$ will be used. Note that $J^{(1)} \geqslant 0$ due to the structure of Eq. (10), thereby implying that there is a relationship between the two norm-square forces.

We desire that the reduced space represent the low frequency dynamics of the system below some cutoff frequency value $\sqrt{\gamma^{c}}$. Thus, we define a supplementary cost function $J^{(2)}$ given by 


$$
J^{(2)}=\left\{\begin{array}{l}
0 \quad \text { for } \gamma<\gamma^{c}, \\
\left(\gamma-\gamma^{c}\right) \text { for } \gamma>\gamma^{c},
\end{array}\right.
$$

where $\gamma$ is the sum of all the eigenvalues of the Hessian $\mathbf{G}$ averaged over the subspace volume $\Omega_{z}$,

$$
\gamma=\frac{1}{\Omega_{z}} \int \operatorname{Tr}(\mathbf{G}) d^{M} \mathbf{z} .
$$

Here $\operatorname{Tr}$ is the trace operation and $\mathbf{G}$ is a $M \times M$ matrix whose $i j$ th element is defined as

$$
G_{i j}(\mathbf{z})=\left.\frac{\partial^{2} V(\mathbf{q})}{\partial z_{i} \partial z_{j}}\right|_{\mathbf{q}=\mathbf{P z}} .
$$

This can be rewritten in terms of the elements of the matrix $\mathbf{P}$ as

$$
G_{i j}(\mathbf{z})=\sum_{l, l^{\prime}} P_{l j} P_{l^{\prime} i} H_{l l^{\prime}}(\mathbf{z}) .
$$

Defining $\lambda_{i}^{q}$ and $\lambda_{i}^{z}$ as the $i$ th eigenvalue of the matrix $\mathbf{H}$ and G, respectively, it is shown in Appendix B that a relation exists between these eigenvalues. The linear cost, $\gamma-\gamma^{c}$, in Eq. (12) for $\gamma>\gamma^{c}$ biases the subspace to contain just the low frequency dynamics. The full cost function is

$$
J=J^{(1)}+\alpha J^{(2)},
$$

where $\alpha$ is a positive weight factor chosen properly so as to allow for flexibility in balancing the contributions of $J^{(1)}$ and $J^{(2)}$ to $J$. Minimization of $J$ with respect to the elements of the matrix $\mathbf{P}$ will yield the best attainable subspace consistent with the desired dynamics. We have used a genetic algorithm $^{37}$ to minimize the cost function [Eq. (16)], although other search techniques could be used as well.

\section{ILLUSTRATION WITH A MODEL POTENTIAL}

For the numerical evaluation we express $J^{(1)}$ and $J^{(2)}$ as follows:

$$
\begin{aligned}
J^{(1)}= & -\sum_{l, l^{\prime}, k} P_{l k} P_{l^{\prime} k} \int h_{l}(\mathbf{P z}) h_{l^{\prime}}(\mathbf{P z}) d^{M} \mathbf{z} \\
& +\sum_{l} \int h_{l}^{2}(\mathbf{P z}) d^{M} \mathbf{z}
\end{aligned}
$$

and $J^{(2)}$ for $\gamma>\gamma^{c}$ is given as

$$
J^{(2)}=\sum_{i, l, l^{\prime}} P_{l i} P_{l^{\prime} i} \int H_{l l^{\prime}}(\mathbf{P z}) d^{M} \mathbf{z}-\gamma^{c},
$$

where $H_{l l}(\mathbf{P z})$ is

$$
H_{l l^{\prime}}(\mathbf{P z})=\left.\frac{\partial^{2} V(\mathbf{q})}{\partial q_{l} \partial q_{l^{\prime}}}\right|_{\mathbf{q}=\mathbf{P} \mathbf{z}} .
$$

The model potential is a polynomial

$$
\begin{aligned}
V(\mathbf{q})= & \sum_{i=1}^{N} \frac{1}{2} K_{i} q_{i}^{2}+\sum_{i, j=1}^{N} K_{i j}^{(1)} q_{i} q_{j} \\
& +\sum_{i, j=1}^{N} K_{i j}^{(3)}\left(q_{i}-a\right)^{2}\left(q_{j}-a\right)^{2}
\end{aligned}
$$

and $N$ is the dimension of the system, $K_{i j}^{(1)}=K_{j i}^{(1)}$ and $K_{i j}^{(3)}$ $=K_{j i}^{(3)}$. The quartic part of the potential describes the anharmonic nature of the system. Although this choice of model potential does not represent any particular molecular system, it has all the elements to explore the subspace method presented here. For numerical purposes the integrals appearing in the cost function [Eq. (17)] were split into harmonic $(\mathrm{H})$ and anharmonic $(\mathrm{AH})$ parts

$$
\begin{aligned}
\int h_{l_{1}}(\mathbf{P z}) h_{l_{2}}(\mathbf{P z}) d^{M} \mathbf{z}= & \left.\int h_{l_{1}}(\mathbf{P z}) h_{l_{2}}(\mathbf{P z})\right|_{H} d^{M} \mathbf{z} \\
& +\left.\int h_{l_{1}}(\mathbf{P z}) h_{l_{2}}(\mathbf{P z})\right|_{A H} d^{M} \mathbf{z} .
\end{aligned}
$$

For simplicity we considered a symmetric reduced space domain, i.e., $-L \leqslant z_{i} \leqslant L, i=1,2, \ldots, M$ which leads to the harmonic and the anharmonic parts as follows:

$$
\begin{aligned}
\left.\int h_{l_{1}} h_{l_{2}}\right|_{H} d^{M} \mathbf{z}= & K_{l_{1}} K_{l_{2}} \int q_{l_{1}} q_{l_{2}} d^{M} \mathbf{z} \\
& +2 K_{l_{1}} \sum_{i} K_{i l_{2}}^{(1)} \int q_{l_{1}} q_{i} d^{M} \mathbf{z} \\
& +2 K_{l_{2}} \sum_{i} K_{i l_{1}}^{(1)} \int q_{l_{2}} q_{i} d^{M} \mathbf{z} \\
& +4 \sum_{i i^{\prime}} K_{i l_{1}}^{(1)} K_{i^{\prime} l_{2}}^{(1)} \int q_{i} q_{i^{\prime}} d^{M} \mathbf{z}
\end{aligned}
$$

and

$$
\begin{aligned}
\left.\int h_{l_{1}} h_{l_{2}}\right|_{A H} d^{M} \mathbf{z}= & 4 K_{l_{1}} \sum_{i} K_{i l_{2}}^{(3)} \int\left(q_{i}^{2} q_{l_{1}} q_{l_{2}}+a^{2} q_{l_{1}} q_{l_{2}}+2 a^{2} q_{l_{1}} q_{i}\right) d^{M} \mathbf{z}+8 \sum_{i i^{\prime}} K_{i l_{1}}^{(1)} K_{i^{\prime} l_{2}}^{(3)} \int\left(q_{i^{\prime}}^{2} q_{i} q_{l_{2}}+2 a^{2} q_{i} q_{i^{\prime}}\right. \\
& \left.+a^{2} q_{i} q_{l_{2}}\right) d^{M} \mathbf{z}+4 K_{l_{2}} \sum_{i} K_{i l_{1}}^{(3)} \int\left(q_{i}^{2} q_{l_{2}} q_{l_{1}}+a^{2} q_{l_{2}} q_{l_{1}}+2 a^{2} q_{l_{2}} q_{i}\right) d^{M} \mathbf{z}+8 \sum_{i i^{\prime}} K_{i^{\prime} l_{2}}^{(1)} K_{i l_{1}}^{(3)} \int\left(q_{i}^{2} q_{i^{\prime}} q_{l_{1}}\right. \\
& \left.+2 a^{2} q_{i^{\prime}} q_{i}+a^{2} q_{i^{\prime}} q_{l_{1}}\right) d^{M} \mathbf{z}+16 \sum_{i j} K_{i l_{1}}^{(3)} K_{j l_{2}}^{(3)} \int\left(q_{i}^{2} q_{j}^{2} q_{l_{1}} q_{l_{2}}+a^{2} q_{i}^{2} q_{j}^{2}+2 a^{2} q_{i}^{2} q_{j} q_{l_{1}}+2 a^{2} q_{i}^{2} q_{j} q_{l_{2}}\right. \\
& +a^{2} q_{i}^{2} q_{l_{1}} q_{l_{2}}+a^{4} q_{i}^{2}+2 a^{2} q_{i} q_{l_{1}} q_{j}^{2}+2 a^{2} q_{i} x_{j}^{2} q_{l_{2}}+4 a^{2} q_{i} q_{j} q_{l_{1}} q_{l_{2}}+4 a^{4} q_{i} q_{j}+2 a^{4} q_{i} q_{l_{1}}+2 a^{4} q_{i} q_{l_{2}} \\
& \left.+a^{2} q_{j}^{2} q_{l_{1}} q_{l_{2}}+a^{4} q_{j}^{2}+2 a^{4} q_{j} q_{l_{1}}+2 a^{4} q_{j} q_{l_{2}}+a^{4} q_{l_{1}} q_{l_{2}}+a^{6}\right) d^{M} \mathbf{z} .
\end{aligned}
$$


The integral $\int H_{l l^{\prime}}(\mathbf{z}) d^{M} \mathbf{z}$ in Eq. (18) is given by

$$
\begin{aligned}
\int H_{l l^{\prime}}(\mathbf{P z}) d^{M} \mathbf{z}= & \left(K_{l} \delta_{l l^{\prime}}+K_{l^{\prime} l}^{(1)}+K_{l l^{\prime}}^{(1)}\right) \int d^{M} \mathbf{z} \\
& +8 K_{l l^{\prime}}^{(3)} \int\left(q_{l} q_{l^{\prime}}+a^{2}\right) d^{M} \mathbf{z} \\
& +4 \delta_{l l^{\prime}} \sum_{j} K_{j l}^{(3)}\left(q_{j}^{2}+a^{2}\right) d^{M} \mathbf{z} .
\end{aligned}
$$

Substituting $q_{l}=\Sigma_{m} P_{l m} z_{m}$ we obtain the different integrals in terms of the $\mathbf{P}$ matrix elements and $\mathbf{z}$ vector components (see Appendix $\mathrm{C}$ for the particular cases of subspaces with dimensions $M=2$ and $M=3$ ).

\section{RESULTS AND DISCUSSION}

\section{A. Determining the subspace}

The numerical steps involved in finding the subspace are as follows:

(1) Provide $N$ and $M$, i.e., the dimension of the original space and the subspace. M may ultimately be fixed iteratively to meet desired dynamical criteria. Choose the z-space volume (e.g., specify the value of $L$ ).

(2) Provide initial guessed or estimated values for the elements of the $\mathbf{P}$ matrix. In the present case the initial values were chosen randomly with the constraint that the columns of $\mathbf{P}$ represent orthonormal vectors. If necessary orthonormality may be assured through orthonormalization by the Grahm-Schmidt method.

(3) Provide the frequency cutoff value, $\gamma^{c}$.

(4) Evaluate all the integrals, either numerically or analytically and calculate the cost function J. The genetic algorithm requires only the evaluation of the cost function, whereas other search methods will usually require both the cost function as well as its gradient.

(5) Use the genetic algorithm to find the optimal transformation. ${ }^{37}$ In the present test case a single subspace $\Omega_{z}$ was explored, although a general algorithm would set up additional subspaces to follow the subsequent dynamics. In the present work the quality of the subspace dynamics was tested by following trajectories in the subspace and comparing their behavior to those generated in the full space.

\section{B. Testing the quality of the reduced dynamics}

This testing procedure involved the following steps for the reduced space dynamics.

(a) Provide the initial conditions, $\mathbf{q}\left(t_{0}\right)$ and $\dot{\mathbf{q}}\left(t_{0}\right)$.

(b) Calculate the initial conditions in the reduced space, $\mathbf{z}\left(t_{0}\right)=\mathbf{P}^{T} \mathbf{q}\left(t_{0}\right)$ and $\dot{\mathbf{z}}\left(t_{0}\right)=\mathbf{P}^{T} \dot{\mathbf{q}}\left(t_{0}\right)$.

(c) The position, velocity and acceleration in the reduced space at time $t=t_{0}+\Delta t$ were obtained as follows:

$$
\begin{aligned}
& \mathbf{z}\left(t_{0}+\Delta t\right)=\mathbf{z}\left(t_{0}\right)+\dot{\mathbf{z}}\left(t_{0}\right) \Delta t+\frac{1}{2} \ddot{\mathbf{z}}\left(t_{0}\right) \Delta t^{2}, \\
& \dot{\mathbf{z}}\left(t_{0}+\Delta t / 2\right)=\dot{\mathbf{z}}\left(t_{0}\right)+1 / 2 \ddot{\mathbf{z}}\left(t_{0}\right) \Delta t, \\
& \mathbf{q}\left(t_{0}+\Delta t\right)=\mathbf{P} \mathbf{z}\left(t_{0}+\Delta t\right),
\end{aligned}
$$

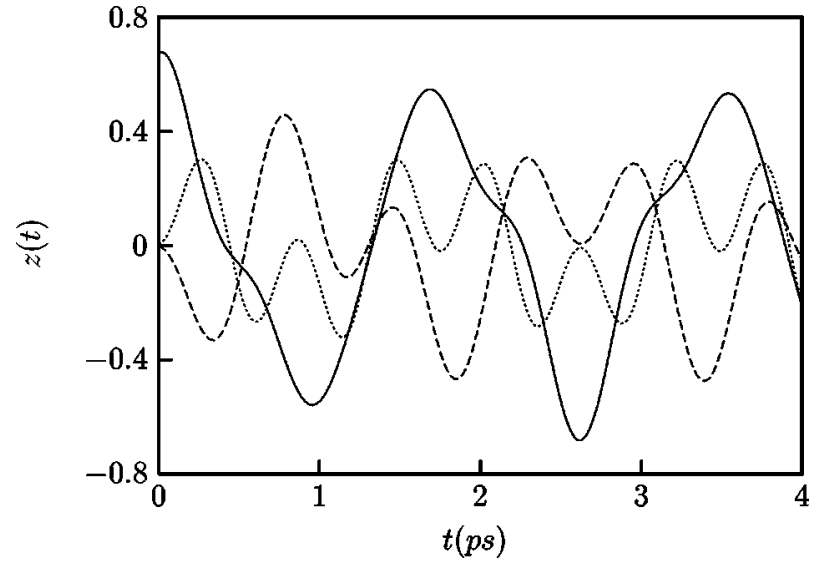

FIG. 1. Trajectories of the subspace coordinates $z_{1}$ (solid lines), $z_{2}$ (dashed lines) and $z_{3}$ (dotted lines) for a linear coupled system. The parameters are $N=6, \quad M=3, \quad K_{1}=10, \quad K_{2}=0.3, \quad K_{3}=14, \quad K_{4}=12, \quad K_{5}=0.1, \quad K_{6}$ $=0.2,(\mathrm{kcal} / \mathrm{mol}) / \AA^{2}, \quad K_{15}^{(1)}=K_{35}^{(1)}=K_{45}^{(1)}=K_{16}^{(1)}=K_{36}^{(1)}=K_{46}^{(1)}=K_{12}^{(1)}=K_{23}^{(1)}$ $=K_{24}^{(1)}=0.2(\mathrm{kcal} / \mathrm{mol}) / \AA^{2}, K_{i j}^{(3)}=0$ and $\gamma^{c}=0.8(\mathrm{kcal} / \mathrm{mol}) / \AA^{2}$.

$$
\begin{aligned}
& \ddot{z}_{j}\left(t_{0}+\Delta t\right)=\left.\sum_{i=1}^{N} P_{i j} \frac{\partial V(\mathbf{q})}{\partial q_{i}}\right|_{\mathbf{q}=\mathbf{q}\left(t_{0}+\Delta t\right)}, \\
& \dot{\mathbf{z}}\left(t_{0}+\Delta t\right)=\dot{\mathbf{z}}\left(t_{0}+\Delta t / 2\right)+1 / 2 \ddot{\mathbf{z}}\left(t_{0}+\Delta t\right) \Delta t .
\end{aligned}
$$

This constitutes the velocity verlet scheme for the reduced equation of motion.

(d) Calculate the position variables $\mathbf{q}$ in the full space at time $t=t_{0}+\Delta t$ by the back transform, $\mathbf{q}\left(t_{0}+\Delta t\right)$ $=\mathbf{P z}\left(t_{0}+\Delta t\right)$.

(e) Repeat steps $c$ and $d$ until the trajectory $\mathbf{q}(t)$ evolves for a suitable duration of time. For a valid test of performance, the subspace dynamics must remain within the subspace during the full trajectory.

For comparison, we solved the exact trajectories $\mathbf{q}(t)$ from Eq. (1) with the initial conditions $\mathbf{q}\left(t_{0}\right)$ and $\dot{\mathbf{q}}\left(t_{0}\right)$. Figures 1-7 present the results including the trajectories as well as their time- and space-averaged properties.

(A) Linear coupled system: Here we desire to create a $M=3$ dimensional subspace from a $N=6$ dimensional full space. The force constants were arbitrarily chosen as $K_{1}$ $=10, \quad K_{2}=0.3, \quad K_{3}=14, \quad K_{4}=12, \quad K_{5}=0.1, \quad K_{6}$ $=0.2(\mathrm{kcal} / \mathrm{mol}) / \AA^{2}$. The system has three modes which are of low frequency compared to the other modes. The coupling amongst the different modes occurred by setting $K_{15}^{(1)}, K_{35}^{(1)}$, $K_{45}^{(1)}, K_{16}^{(1)}, K_{36}^{(1)}, K_{46}^{(1)}, K_{12}^{(1)}, K_{23}^{(1)}, K_{24}^{(1)}$ as $0.2 \mathrm{kcal} / \mathrm{mol} / \AA^{2}$ while the other $K_{i j}^{(1)}$ values were zero; all of the nonlinear coupling terms $K_{i j}^{(3)}$ are zero. This is a harmonic system where the low frequency motion of $q_{2}, q_{5}$ and $q_{6}$ are coupled to that of the high frequency motion in the chosen coordinates. We sought the subspace dynamics associated with a mean frequency less than $1.83 \times 10^{13} \mathrm{rad} / \mathrm{s}$ corresponding to $\gamma^{c}=0.8(\mathrm{kcal} / \mathrm{mol}) / \AA^{2}$. This system was chosen to test the ability for the method to automatically identify the subspace that contains the low frequency motion. This system has an exact subspace, but the algorithm did not build in any such knowledge. The subspace with $L=2.0 \AA$, identified 

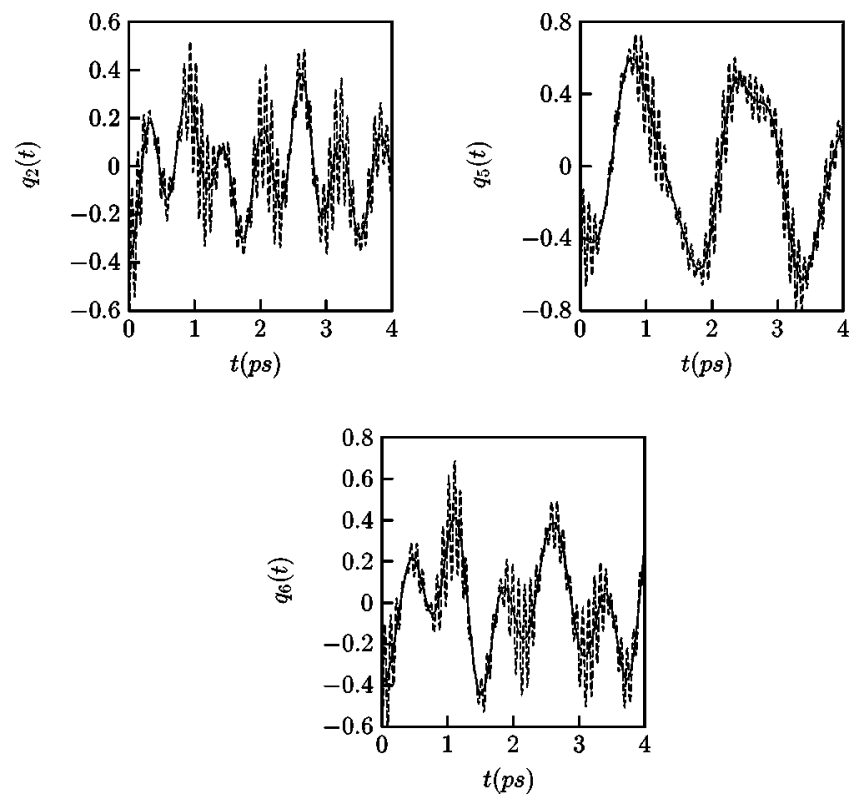

FIG. 2. Low frequency trajectories $q_{2}, q_{5}$, and $q_{6}$ for a linear coupled system, where the solid lines correspond to the subspace results and the broken lines to the exact full space method. The parameters are the same as in Fig. 1.

by the genetic algorithm operation with a population size of 400 gives the $\mathbf{P}$ matrix as

$$
\mathbf{P}=\left(\begin{array}{ccc}
0.072 & 0.000251 & -0.00454 \\
-0.57 & -0.0422 & 0.816 \\
0.0493 & -0.000482 & 0.0031 \\
0.058 & 0.000235 & -0.000574 \\
-0.573 & 0.729 & -0.370 \\
-0.576 & -0.683 & -0.4445
\end{array}\right)
$$
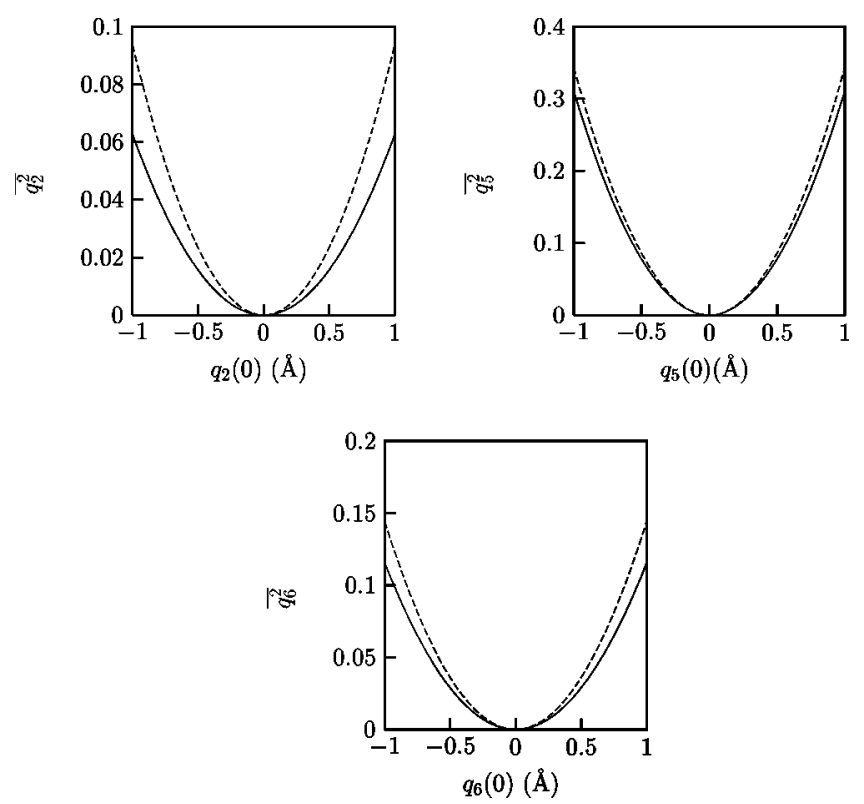

FIG. 3. Time-averaged properties viz., $\overline{q_{2}^{2}}, \overline{q_{5}^{2}}$, and $\overline{q_{6}^{2}}$ as a function of the initial values of $q_{2}(0), q_{5}(0)$, and $q_{6}(0)$ for a linear coupled system. The solid lines correspond to the subspace method and the broken lines to the exact full space dynamical results. The parameters are the same as in Fig. 1.
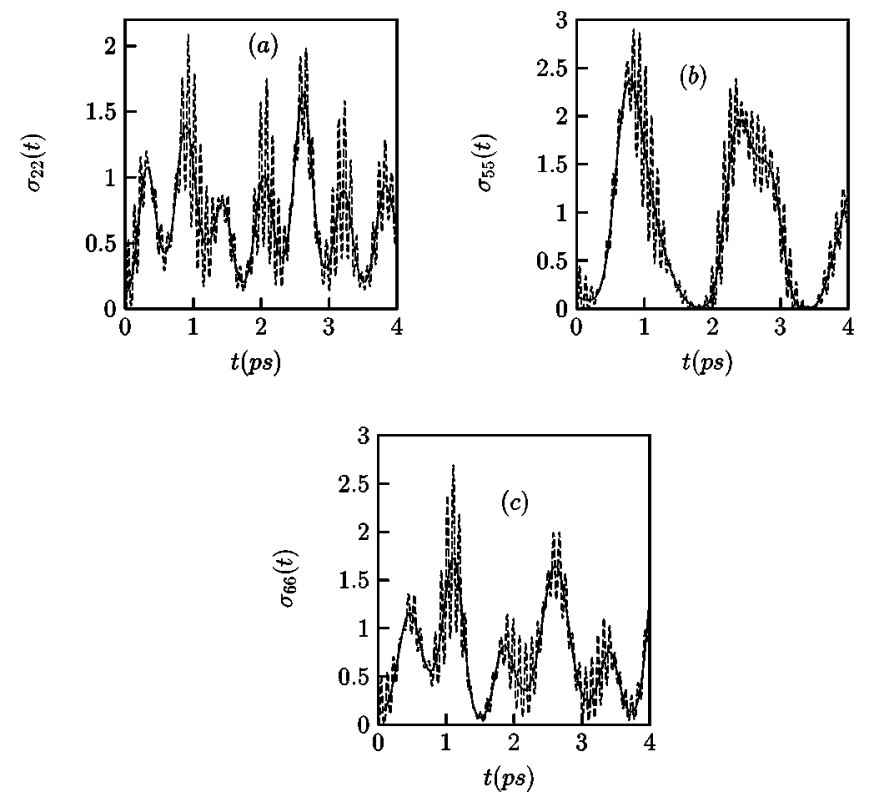

FIG. 4. The space-averaged dynamical properties viz., $\sigma_{22}$ (a) and $\sigma_{55}$ (b) and $\sigma_{66}(\mathrm{c})$ calculated over an ensemble of the initial values of $q_{2}(0), q_{5}(0)$ and $q_{6}(0)$, for a linear coupled system. The solid lines correspond to the subspace method and the broken line to the exact full space dynamical results. The parameters are the same as in Fig. 1.

starting from an initial random orthonormal matrix. A close look at the optimal $\mathbf{P}$ reveals that the columns of the matrix are a linear combination of the $M=3$ lowest frequency eigenvectors of the Hessian in the full space (an analytical proof is given in Appendix D). The dominant role of $q_{2}, q_{5}$, and $q_{6}$ is evident in the transformation. Figure 1 shows the trajectories of the reduced coordinates, $z_{1}, z_{2}$, and $z_{3}$ and Fig. 2 shows the trajectories for $q_{2}, q_{5}$, and $q_{6}$ obtained
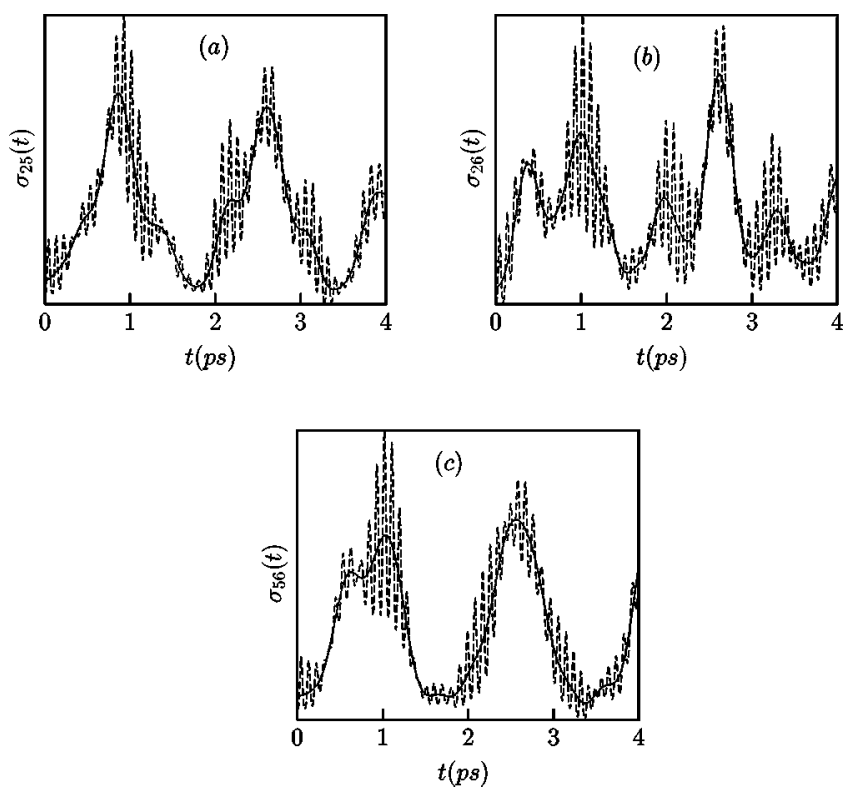

FIG. 5. The space-averaged dynamical properties viz., $\sigma_{25}$ (a) and $\sigma_{26}$ (b) and $\sigma_{56}(\mathrm{c})$ calculated over an ensemble of the initial values $q_{2}(0), q_{5}(0)$ and $q_{6}(0)$, for a linear coupled system. The solid lines correspond to the subspace method and the broken lines to the exact full space dynamical results. The parameters are the same as in Fig. 1. 

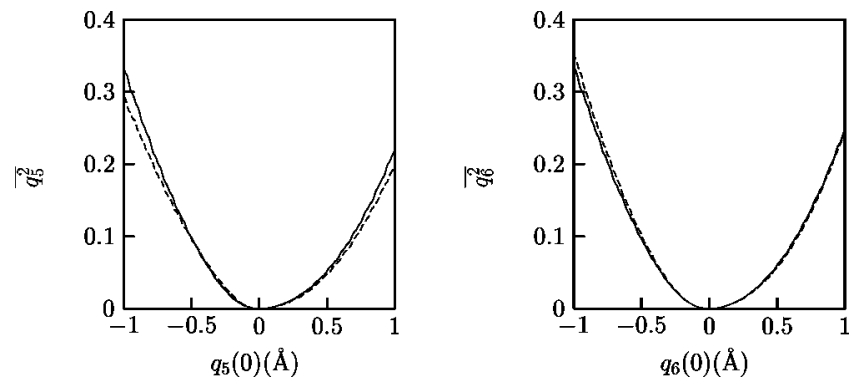

FIG. 6. Time-averaged properties viz., $\overline{q_{5}^{2}}$ and $\overline{q_{6}^{2}}$ as a function of the initial values of $q_{5}(0)$ and $q_{6}(0)$ for a non linear system. The solid lines correspond to the subspace method and the broken lines to the exact full space dynamical results. The parameters are $N=6, M=2, K_{1}=10, K_{2}=11, K_{3}$ $=14, K_{4}=12, K_{5}=0.1, K_{6}=0.2(\mathrm{kcal} / \mathrm{mol}) / \AA^{2}, K_{15}^{(1)}=K_{25}^{(1)}=K_{36}^{(1)}=K_{46}^{(1)}$ $=0.2(\mathrm{kcal} / \mathrm{mol}) / \AA^{2}, \quad K_{i j}^{(3)}=0.01(\mathrm{kcal} / \mathrm{mol}) / \AA^{4} \forall i=1,2, \ldots, N$ and $j$ $=1,2, \ldots, N, a=0.1 \AA$ and $\gamma^{c}=5.5(\mathrm{kcal} / \mathrm{mol}) / \AA^{2}$.

with the subspace method (solid lines) along with the corresponding exact values (broken lines). In calculating the trajectories the initial conditions are $q_{1}(0)=q_{3}(0)=q_{4}(0)$ $=-2.69 \AA$ and $q_{2}(0)=q_{5}(0)=q_{6}(0)=-0.67 \AA$ and $\dot{\mathbf{q}}$ $=0$. The subspace method is able to pick up the low frequency motion underlying $q_{2}, q_{5}$, and $q_{6}$. Notice that all the high frequency motion appearing in the exact full space calculation (broken lines) gets averaged out in the subspace dynamics showing that the subspace method filters out the nonessential parts of the dynamics.

In order to assess the general quality of the subspace a set of space and time averaged properties were calculated. Figure 3 displays the time-averaged quantities $\overline{q_{i}^{2}}$ $=1 / T \int_{0}^{T} q_{i}^{2}(t) d t$ for $i=2,5$, and 6 at different initial values
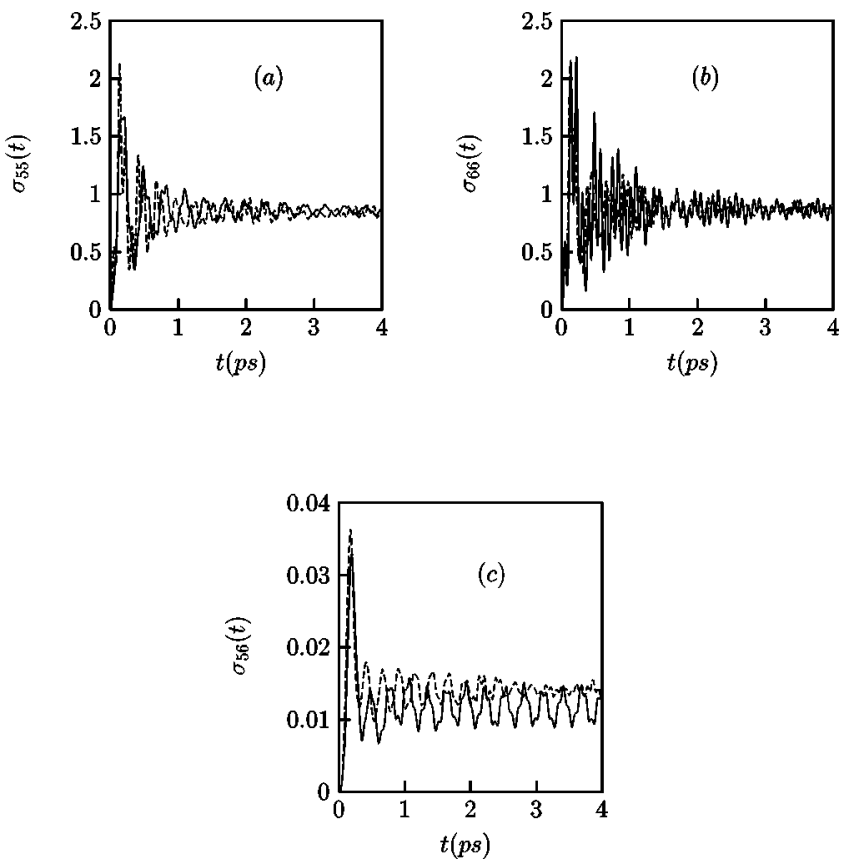

FIG. 7. The space-averaged dynamical properties viz., $\sigma_{55}$ (label (a)) and $\sigma_{66}$ (label (b)) and $\sigma_{56}$ (label (c)) calculated over an ensemble of the initial values of $q_{5}(0)$ and $q_{6}(0)$, for a nonlinear system. The solid lines correspond to the subspace method and the broken lines to the exact full space dynamical results. The parameters are the same as in Fig. 6 .

of $q_{2}(0), q_{5}(0)$, and $q_{6}(0)$, respectively, whereas Figs. 4 and 5 display the space-averaged dynamical variances, viz., $\sigma_{22}$ [label (a) Fig. 4], $\sigma_{55}$ [label (b) Fig. 4], $\sigma_{66}$ [label (c) Fig. 4], $\sigma_{25}$ [label (a) Fig. 5], $\sigma_{26}$ [label (b) Fig. 5], and $\sigma_{56}$ [label (c) Fig. 5] defined as

$$
\sigma_{i j}(t)=\left\{\begin{array}{l}
\iint\left(q_{i}\left(t, q_{i}(0)\right)-q_{i}\left(0, q_{i}(0)\right)\right)\left(q_{j}\left(t, q_{j}(0)\right)-q_{j}\left(0, q_{j}(0)\right)\right) d q_{i}(0) d q_{j}(0) \quad \text { for } i \neq j, \\
\int\left(q_{i}\left(t, q_{i}(0)\right)-q_{i}\left(0, q_{i}(0)\right)\right)^{2} d q_{i}(0) \quad \text { for } i=j .
\end{array}\right.
$$

Figures 3-5 show that the subspace method acts as an effective filter for the high frequency motion over a broad set of conditions. The CPU time for evaluating the matrix $\mathbf{P}$ was 12.9 min and that for calculating one trajectory corresponding to the low frequency motion for 4 ps was 3.9 $\times 10^{-4}$ min whereas the CPU time for calculating one trajectory following exact dynamical calculation was 2.5 $\times 10^{-3}$ min during the same interval of time. The time step was $\Delta t=2.05 \mathrm{fs}$ in the exact dynamical calculation and 24.6 fs in the subspace calculation. Thus, in the subspace calculation of a single trajectory we obtained a reduction in the total time step size by $\approx 1 / 12$ assuming that $\mathbf{P}$ was known. However, the calculation of a single trajectory in the subspace method is roughly 6 times faster than that in the exact method because of the need to evaluate the back transformation $\mathbf{q}=\mathbf{P z}$ and the force $g_{i}(\mathbf{z})$.

(B) Nonlinear system: Here we study a nonlinear system characterized by the parameters $K_{1}=10, K_{2}=11, K_{3}=14$, $K_{4}=12, \quad K_{5}=0.1, \quad K_{6}=0.2(\mathrm{kcal} / \mathrm{mol}) / \AA^{2}, \quad K_{15}^{(1)}=K_{25}^{(1)}$
$=K_{36}^{(1)}=K_{46}^{(1)}=0.2,(\mathrm{kcal} / \mathrm{mol}) / \AA^{2}$ and the nonlinear coupling is chosen as $K_{i j}^{(3)}=0.01(\mathrm{kcal} / \mathrm{mol}) / \AA^{4} \quad \forall i$ $=1,2, \ldots, N$ and $j=1,2, \ldots, N$ and $a=0.1 \AA$. This system was chosen to have a reduced space of dimension $M=2$. We desired that the subspace of size $L=1.5 \AA$, seek out the dynamics such that $\gamma^{c}=5.5(\mathrm{kcal} / \mathrm{mol}) / \AA^{2}$ which corresponds to a frequency cutoff $1.6 \times 10^{14} \mathrm{radian} / \mathrm{s}$. The optimal $\mathbf{P}$ obtained after iterating from a random initial guess was

$$
\mathbf{P}=\left(\begin{array}{cc}
-0.372 & 0.274 \\
-0.458 & 0.404 \\
0.007 & -0.029 \\
-0.487 & 0.419 \\
0.5 & 0.556 \\
0.406 & 0.526
\end{array}\right)
$$

Figures 6 and 7 present the time averaged and space averaged properties for both the subspace method (solid lines) 
and the exact method (broken lines). In both Figs. 6 and 7 the subspace method reproduces the low frequency dynamics in an average manner compared to that of the exact full dynamical calculation. The CPU time for evaluating the matrix $\mathbf{P}$ was $38.9 \mathrm{~min}$ and that for calculating one trajectory corresponding to the low frequency motion for 4 ps was 3.9 $\times 10^{-4}$ min whereas the CPU for calculating one trajectory following the exact dynamics was $3.21 \times 10^{-3}$ min during the same interval of time. The time step was $\Delta t=1.9 \mathrm{fs}$ in the exact dynamical calculation and $19.0 \mathrm{fs}$ in the subspace calculation. Thus, in the subspace calculation of a single trajectory we obtained a reduction in the total time step by $\approx 1 / 10$ over that in the exact calculation assuming $\mathbf{P}$ was known. The calculation of a single trajectory in the subspace method is roughly 8 times faster than that in the exact method, because of the need to evaluate the back transformation $\mathbf{q}=\mathbf{P z}$ and the force $g_{i}(\mathbf{z})$.

\section{SUMMARY AND CONCLUDING REMARKS}

This study presented a new subspace method to treat low frequency classical molecular dynamics. The approach seeks an optimal subspace of dimension $M$ from its parent full space of dimension $N$ such that $M \ll N$ by suitably defining a cost function. A search is performed to identify the optimal transformation relating the $N$ - and $M$-dimensional spaces under the desire to capture the low frequency system motion. The demonstration of the method with a model potential revealed that the dynamics in the subspace acted like a filter for the high frequency motions, automatically determining the essential low frequency motion. The choice of the cutoff frequency $\sqrt{\gamma^{c}}$ plays an important role in this method. From Eq. (12) the cost function $J^{(2)}$ defines the difference between the square of the cutoff frequency and the sum of all the eigenvalues of the subspace Hessian $\mathbf{G}$ averaged over the reduced space. Physically, optimization of $J^{(2)}$ directs the system's dynamics to be below the frequency $\sqrt{\gamma^{c}}$, thereby focusing on the system slow motion. Thus, it is crucial that the subspace dimension, $M$ be consistent with the frequency cutoff. In its full form the approach can be applied to systematically perform long time scale dynamics. The present paper focused on the essential aspects of the algorithm that seeks out a subspace keeping in mind applications to long time scale conformational molecular dynamics. The demonstrations in the paper were chosen with simple model systems to test the optimal subspace method. Future applications of the method would be for polyatomic molecules, including those of biological relevance with significant long time behavior.

The subspace method differs from the constrained molecular dynamics method where degrees of freedom are eliminated through the introduction of holonomic constraints so that the associated momenta are zero. However, under realistic conditions, no matter how stiff some of these degrees of freedom (bond lengths, bond angles, etc.) may be, the associated momenta are not zero. At thermal equilibrium, there is a Boltzmann distribution of these momenta, and the constrained dynamics can differ significantly from the true behavior. This can result in the constrained dynamical trajec- tories covering a different region of phase space than the true trajectories. ${ }^{38}$ The subspace method, in this regard, does not force any coordinates to be irrelevant and the associated conjugate momenta are not set to zero, since generally $P_{l_{c} m}$ $\neq 0$ where $l_{c}=1, \ldots, N-M$ represent the normally constrained coordinates. Furthermore, the parameter $\gamma_{c}$ could be relaxed to take on a higher value so that some degrees of high-frequency contributions can be brought back into the low-frequency dynamics. Thus, the subspace method is more flexible than the constrained dynamics method. In this regard, the method can automatically identify the important slow structural degrees of freedom. A full algorithm would identify successive subspaces following the dynamics along in much the same way done before ${ }^{33,34}$ where the subspace was defined by the local Hessians. The present method is more flexible and a further generalization will be presented elsewhere permitting a larger subspace by allowing for $\mathbf{P}$ to depend on $\mathbf{z} .{ }^{39}$ This would better account for the equilibrium averages associated with the trajectories than the usual constrained dynamics method.

\section{ACKNOWLEDGMENT}

The authors acknowledge support from the ARO.

\section{APPENDIX A: THE COST FUNCTION}

This Appendix simplifies the cost function in Eq. (10) to yield Eq. (11). The integrand in Eq. (10) can be rewritten as

$$
\|\mathbf{P} \ddot{\mathbf{z}}+\mathbf{h}(\mathbf{q})\|^{2}=\|\mathbf{P} \ddot{\mathbf{z}}\|^{2}+\|\mathbf{h}(\mathbf{q})\|^{2}+2\langle\mathbf{P} \ddot{\mathbf{z}}, \mathbf{h}(\mathbf{q})\rangle,
$$

where the first term on the right-hand side is given by

$$
\begin{aligned}
\|\mathbf{P} \ddot{\mathbf{z}}\|^{2} & =\sum_{l=1}^{N}\left(\sum_{k=1}^{M} P_{l k} \ddot{z}_{k}\right)^{2}=\sum_{l, k, k^{\prime}} P_{l k} P_{l k^{\prime}} \ddot{z}_{k} \ddot{z}_{k^{\prime}}=\sum_{k=1}^{M} \ddot{z}_{k}^{2} \\
& =\|\ddot{\mathbf{z}}\|^{2}=\|\mathbf{g}(\mathbf{z})\|^{2}
\end{aligned}
$$

and the third term is given by

$$
2\langle\mathbf{P} \ddot{\mathbf{z}}, \mathbf{h}(\mathbf{q})\rangle=2 \sum_{l}(\mathbf{P} \ddot{\mathbf{z}})_{l} h_{l}(\mathbf{q})=2 \sum_{l, k} P_{l k} \ddot{z}_{k} h_{l}(\mathbf{q}) .
$$

Now $g_{k}(\mathbf{z})$ can be written as

$$
g_{k}(\mathbf{z})=\sum_{l^{\prime}} h_{l^{\prime}}(\mathbf{q}) \frac{\partial q_{l^{\prime}}}{\partial z_{k}} .
$$

Since we assume that $q_{l^{\prime}}=\Sigma_{k^{\prime}} P_{l^{\prime} k^{\prime}} z_{k^{\prime}}$, we get $\partial q_{l^{\prime}} / \partial z_{k}$ $=\Sigma_{k^{\prime}} P_{l^{\prime} k^{\prime}} \delta_{k k^{\prime}}=P_{l^{\prime} k}$. This simplifies $g_{k}(\mathbf{z})$ to

$$
g_{k}(\mathbf{z})=\sum_{l^{\prime}} P_{l^{\prime} k} h_{l^{\prime}}(\mathbf{q}) .
$$

We obtain using Eq. (6),

$$
2\langle\mathbf{P} \ddot{\mathbf{z}}, \mathbf{h}(\mathbf{q})\rangle=-2 \sum_{k} \ddot{z}_{k}^{2}=-2\|\mathbf{g}(\mathbf{z})\|^{2},
$$

and this gives

$$
\|\mathbf{P} \ddot{\mathbf{z}}+\mathbf{h}(\mathbf{q})\|^{2}=\|\mathbf{h}(\mathbf{q})\|^{2}-\|\mathbf{g}(\mathbf{z})\|^{2},
$$

to finally yield 


$$
J^{(1)}=\int\left[\|\mathbf{h}(\mathbf{q})\|^{2}-\|\mathbf{g}(\mathbf{z})\|^{2}\right] d^{M} \mathbf{z} .
$$

\section{APPENDIX B: RELATION BETWEEN THE EIGENVALUES IN SUBSPACE AND FULL SPACE}

In this Appendix we provide a relation between the eigenvalues in the subspace and the full space. In particular we show that for a harmonic potential the sum of the associated $M$ eigenvalues are equal in both the spaces whereas for an anharmonic potential the sum of the $M$ eigenvalues in the subspace is a linear combination of all the eigenvalues in the full space. From Eq. (15) we can write

$$
\sum_{i=1}^{M} \lambda_{i}^{z}=\sum_{i=1}^{M} G_{i i}=\sum_{l, l^{\prime}} P_{l i} P_{l^{\prime} i} H_{l l^{\prime}} .
$$

Defining $Q$ as the eigenvector matrix of the full space Hessian $H=Q \Lambda^{q} Q^{T}$ where $\Lambda^{q}$ is diagonal with eigenvalues $\lambda_{j}^{q}$, $j=1, \ldots, N$ we get

$$
H_{l l^{\prime}}=\sum_{m=1}^{N} Q_{l m} Q_{l^{\prime} m} \lambda_{m}^{q} .
$$

Substituting into Eq. (B1) we obtain

$$
\sum_{i=1}^{M} \lambda_{i}^{z}=\sum_{l, l^{\prime}, i, m} P_{l i} P_{l^{\prime} i} Q_{l m} Q_{l^{\prime} m} \lambda_{m}^{q} .
$$

In general, the columns of the $\mathbf{P}$ matrix are the linear superposition of the $N$ eigenvectors of the full space Hessian matrix,

$$
P_{l i}=\sum_{i^{\prime}=1}^{N} \alpha_{i i^{\prime}} Q_{l i^{\prime}} .
$$

Thus we obtain the general relation between $\lambda_{i}^{z}$ and $\lambda_{j}^{q}$ where $i$ runs from 1 to $M$ and $j$ from 1 to $N$,

$$
\sum_{i=1}^{M} \lambda_{i}^{z}=\sum_{l, l^{\prime}, i, m, k, j} \alpha_{i k} \alpha_{i j} Q_{l k} Q_{l^{\prime} j} Q_{l m} Q_{l^{\prime} m} \lambda_{m}^{q} .
$$

Since the eigenvectors of the $Q$ matrix represent an orthonormal basis we immediately obtain

$$
\sum_{i=1}^{M} \lambda_{i}^{z}=\sum_{i, m}^{M, N} \alpha_{i m}^{2} \lambda_{m}^{q} .
$$

The subspace eigenvalues are a particular linear combination of the full space eigenvalues. For a harmonic potential the columns of the $\mathbf{P}$ matrix are those of the $Q$ matrix in which case $\sum_{i=1}^{M} \lambda_{i}^{z}=\sum_{m=1}^{M} \lambda_{m}^{q}$.

\section{APPENDIX C: INTEGRALS IN EVALUATING THE REDUCED SPACE EQUATIONS}

In this Appendix we provide the analytical expressions for the integrals appearing in Eqs. (22)-(24) for two cases viz., $M=2$ and $M=3$. For reduced space dimension $M=2$ we have

$$
\begin{aligned}
& \int d^{M} \mathbf{z}=4 L^{2}, \\
& \int q_{l} q_{l^{\prime}} d^{M} \mathbf{z}=\frac{4}{3} L^{4} \sum_{m} P_{l m} P_{l^{\prime} m},
\end{aligned}
$$

For $M=3$ we have

$$
\begin{aligned}
& \int d^{M} \mathbf{z}=8 L^{3}, \\
& \int q_{l} q_{l^{\prime}} d^{M} \mathbf{z}=\frac{8}{3} L^{5} \sum_{m} P_{l m} P_{l^{\prime} m} .
\end{aligned}
$$

\section{APPENDIX D: THE SUBSPACE TRANSFORMATION FOR A HARMONIC SYSTEM}

In this Appendix we prove that the cost function $J^{(1)}$ $=0$ for harmonic potential when the columns of the $\mathbf{P}$ matrix can be represented as a linear superposition of $M$ eigenvectors of the Hessian. In general, any column of $\mathbf{P}$ is a linear superposition of $N$ eigenvectors of the full space Hessian,

$$
P_{i j}=\sum_{k=1}^{N} c_{k j} Q_{i k}
$$

such that

$$
\sum_{k=1}^{N} c_{k j} c_{k j^{\prime}}=\delta_{j j^{\prime}},
$$


where $Q_{i k}$ is the $i k$ th element of the eigenvector matrix $\mathbf{Q}$ that diagonalizes the full space, i.e.,

$$
\begin{aligned}
& \mathbf{Q}^{T} \mathbf{H} \mathbf{Q}=\lambda, \\
& \mathbf{H} \mathbf{Q}^{(j)}=\lambda_{j}^{q} \mathbf{Q}^{(j)},
\end{aligned}
$$

where $\mathbf{Q}^{(j)}$ represents the $j$ th column of the matrix $\mathbf{Q}$. This gives

$$
\sum_{k=1}^{N} H_{l k} Q_{k j}=\lambda_{j}^{q} Q_{l j}
$$

The Harmonic case can be written as

$$
h_{l}=\sum_{i=1}^{N} H_{i l} q_{i} \text {. }
$$

Writing $J^{(1)}=J_{1}^{(1)}-J_{2}^{(1)}$ where $J_{1}^{(1)}=\Sigma_{l} \int h_{l}^{2}(\mathbf{z}) d \mathbf{z}$ and $J_{2}^{(1)}$ $=\Sigma_{l, l^{\prime}, k} P_{l k} P_{l^{\prime} k} \int h_{l}(\mathbf{z}) h_{l^{\prime}}(\mathbf{z}) d \mathbf{z}$ we obtain

$$
J_{1}^{(1)}=\int \sum_{l, m, m^{\prime}, k, k^{\prime}} \lambda_{k}^{q} \lambda_{k^{\prime}}^{q} Q_{l k} Q_{l k^{\prime}} c_{k m} c_{k^{\prime} m^{\prime}} z_{m} z_{m^{\prime}} d \mathbf{z} .
$$

Since the columns of $\mathbf{Q}$ are orthonormal, i.e., $\Sigma_{l} Q_{l k} Q_{l k^{\prime}}$ $=\delta_{k k^{\prime}}$ we get

$$
J_{1}^{(1)}=\sum_{m, m^{\prime}, k, k^{\prime}} \lambda_{k}^{q} \lambda_{k^{\prime}}^{q} c_{k m} c_{k^{\prime} m^{\prime}} \delta_{k k^{\prime}} \int z_{m} z_{m^{\prime}} d \mathbf{z} .
$$

Similarly the expression for $J_{2}^{(1)}$ can be simplified as

$$
J_{2}^{(1)}=\sum_{m, m^{\prime}, k, k^{\prime}, s} \lambda_{k}^{q} \lambda_{k^{\prime}}^{q} c_{k m} c_{k^{\prime} m^{\prime}} c_{k s} c_{k^{\prime} s} \int z_{m} z_{m^{\prime}} d \mathbf{z} .
$$

We obtain

$$
\begin{aligned}
J^{(1)}= & -\sum_{m, m^{\prime}, k, k^{\prime}} \lambda_{k}^{q} \lambda_{k^{\prime}}^{q} c_{k m} c_{k^{\prime} m^{\prime}} \int z_{m} z_{m^{\prime}} d \mathbf{z} \\
& \times \sum_{s=1}^{M} c_{k s} c_{k^{\prime} s}-\delta_{k k^{\prime}} .
\end{aligned}
$$

Thus, $\quad J^{(1)}=0$ if $\sum_{s=1}^{M} c_{k s} c_{k^{\prime} s}=\delta_{k k^{\prime}}=\sum_{m} c_{m k} c_{m k^{\prime}}$. That means the matrix $\mathbf{C}$ should have the property $\mathbf{C C}^{T}=\mathbf{C}^{T} \mathbf{C}$ $=\mathbf{I}_{M}$, where $\mathbf{I}_{M}$ is an $M \times M$ unit matrix. The columns of the matrix $\mathbf{P}$ are a linear combination of $M$ eigenvectors of the full space Hessian.

${ }^{1}$ C. L. Brooks, M. Karplus, and B. M. Pettitt, Proteins: A Theoretical Perspective of Dynamics, Structure and Thermodynamics (WileyInterscience, New York, 1988).
${ }^{2}$ M. Karplus, Modeling of Molecular Structures and Properties, edited by J. L. Rivail (Elsevier Science, Amsterdam, 1990), p. 427.

${ }^{3}$ Y. X. Chang and K. F. Freed, J. Chem. Phys. 99, 8016 (1993).

${ }^{4}$ A. M. Nyberg and T. Schlick, Chem. Phys. Lett. 198, 538 (1992).

${ }^{5}$ B. R. Brooks, D. Janezic, and M. Karplus, J. Comput. Chem. 16, 1522 (1995).

${ }^{6}$ D. A. Case, Curr. Opin. Struct. Biol. 4, 285 (1994).

${ }^{7}$ C. Schutte, A. Fischer, W. Huisinga, and P. Deuflhard, J. Comput. Phys. 151, 146 (1999).

${ }^{8}$ T. Ichiye and M. Karplus, Proteins 11, 205 (1991).

${ }^{9}$ A. Kitao, F. Hirata, and N. Go, Chem. Phys. 158, 447 (1991).

${ }^{10}$ A. E. Garcia, Phys. Rev. Lett. 68, 2696 (1992).

${ }^{11}$ L. Sirovich and J. D. Rodriguez, Phys. Lett. A 120, 211 (1987).

${ }^{12}$ D. H. Lu and W. L. Hase, J. Chem. Phys. 89, 6723 (1988).

${ }^{13}$ A. Kuppermann, J. Phys. Chem. 83, 171 (1979).

${ }^{14}$ Q. Sun and J. M. Bowman, J. Chem. Phys. 92, 1021 (1990).

${ }^{15}$ J. M. Bowman, Adv. Chem. Phys. 61, 115 (1985).

${ }^{16}$ Q. Sun and J. M. Bowman, Int. J. Quantum Chem., Quantum Chem. Symp. 23, 115 (1989).

${ }^{17}$ J. P. Ryckaert, G. Ciccotti, and H. J. C. Berendsen, J. Comput. Phys. 23, 327 (1977).

${ }^{18}$ S. Miyamoto and P. A. Kollman, J. Comput. Chem. 13, 952 (1992).

${ }^{19}$ D. J. Tobias and C. L. Brooks, J. Chem. Phys. 89, 5115 (1988).

${ }^{20}$ S. G. Lambrakos and M. Peyrard, J. Chem. Phys. 93, 4329 (1990).

${ }^{21}$ Y. Qin and D. L. Thompson, J. Chem. Phys. 96, 1992 (1992).

${ }^{22}$ A. Amadei, A. B. M. Linssen, and H. J. C. Berendsen, Proteins 17, 412 (1993).

${ }^{23}$ T. Horiuchi and N. Go, Proteins 10, 106 (1991).

${ }^{24}$ D. M. F. Van Aalten, A. Amadei, A. B. M. Linssen, V. G. H. Eijsink, G. Vriend, and H. J. C. Berendsen, Proteins 22, 45 (1995).

${ }^{25}$ M. A. Balsera, W. Wriggers, Y. Oono, and K. Schulten, J. Phys. Chem. 100, 2567 (1996).

${ }^{26}$ S. C. Phillips, J. W. Essex, and C. M. Edge, J. Chem. Phys. 112, 2586 (2000).

${ }^{27}$ R. B. Sessions, P. D. Osguthorpe, and D. J. Osguthorpe, J. Mol. Biol. 210, 617 (1989).

${ }^{28}$ P. D. Osguthorpe, C. M. Maunder, and D. J. Osguthorpe, J. Comput.Aided Mol. Des. 10, 177 (1996).

${ }^{29}$ M. Levitt, J. Mol. Biol. 220, 1 (1991).

${ }^{30}$ A. Askar, A. E. Cetin, and H. Rabitz, J. Phys. Chem. 100, 19165 (1996).

${ }^{31}$ H. Lu, B. Isralewitz, A. Krammer, V. Vogel, and K. Schulten, Biophys. J. 75, 662 (1998).

${ }^{32}$ C. Schutte and F. A. Bornemann, Nonlinear Analysis-Theory, Method Applications 30, 1805 (1997).

${ }^{33}$ B. Space, H. Rabitz, and A. Askar, J. Chem. Phys. 99, 9070 (1993).

${ }^{34}$ A. Askar, B. Space, and H. Rabitz, J. Phys. Chem. 99, 7330 (1995).

${ }^{35}$ K. Willcox and J. Peraire, AIAA J. 40, 2323 (2002).

${ }^{36}$ K. Willcox, J. Paduano, J. Peraire, and K. Hall, AIAA Paper 99-1467, Proceedings of 40th AIAA Structures, Structural Dynamics and Materials Conference, 1999.

${ }^{37}$ S. R. Ladd, Genetic Algorithms in $\mathrm{C}++$ (M and T Books, New York, 1996); D. E. Goldberg, Genetic Algorithms in Search, Optimization and Machine Learning (Addison-Wesley, New York, 1989).

${ }^{38}$ N. Go and H. A. Scheraga, Macromolecules 9, 535 (1976).

${ }^{39}$ B. K. Dey, H. Rabitz, and A. Askar (unpublished). 